\title{
Prospects and requirements for an operational modelling unit in flood crisis situations
}

\author{
Katharina Anders ${ }^{1}$, André Assmann ${ }^{1, a}$, and Kathrina Fritsch ${ }^{1}$ \\ ${ }^{1}$ geomer GmbH, Im Breitspiel 11B, 69126 Heidelberg, Germany
}

\begin{abstract}
Dike failure events pose severe flood crisis situations on areas in the hinterland of dikes. In recent decades the importance of being prepared for dike breaches has been increasingly recognized. However, the pre-assessment of inundation resulting from dike breaches is possible only based on scenarios, which might not reflect the situation of a real event. This paper presents a setup and workflow that allows to model dike breachinduced inundation operationally, i.e. when an event is imminent or occurring. A comprehensive system setup of an operational modelling unit has been developed and implemented in the frame of a federal project in Saxony-Anhalt, Germany. The modelling unit setup comprises a powerful methodology of flood modelling and elaborated operational guidelines for crisis situations. Nevertheless, it is of fundamental importance that the modelling unit is instated prior to flood events as a permanent system. Moreover the unit needs to be fully integrated in flood crisis management. If these crucial requirements are met, a modelling unit is capable of fundamentally supporting flood management with operational prognoses of adequate quality even in the limited timeframe of crisis situations.
\end{abstract}

\section{Introduction}

Severe flood crisis situations arise from the incidence of catastrophic, mostly unpredicted events. This applies particularly to the occurrence of dike failure, which constitutes a highly unique flood situation characterized primarily by its unpredictable breach location, high flow velocities and the resulting inundation of large areas in rather short time frames.

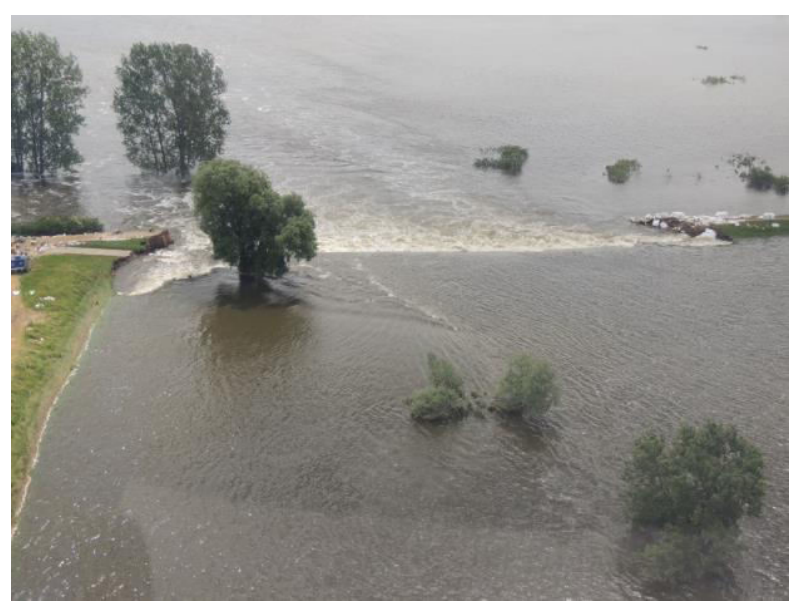

Figure 1. Dike breach on the river Elbe near Fischbeck, June 2013. Photo: Robert Jüpner.

The hazard of dike failure, thus, poses a significant risk on human lives and activity in the hinterland of dikes - areas where people are usually feeling safe from the hazard of inundation given the protection from floods by dike constructions. Nonetheless, it is economically not feasible to provide absolute security by dikes, which are generally built to a certain design standard. This makes respective areas vulnerable towards the inundation resulting from dike breaches, which might occur for numerable reasons, e.g. if the loading by the flood exceeds the design a dike section is constructed for. Furthermore, large stretches of dikes in many parts of Europe are not built in state-of-the-art technology and might fail due to insufficient stability even during less extreme floods $[1,2]$.

Numerous events in recent decades have not only raised social awareness of the possibility of dike breaches. The importance of considering dike breaches in respect of the resulting inundation as well as their influence on flood development has become increasingly recognized by communities and governmental agencies. This is linked to the general change in the thinking of flood management, which was directed from aiming at absolute safety in flood protection to the assessment of given risk and dealing with the consequences of inundation. This risk-based approach is expressed in the EU flood directive on the assessment and management of flood risk [3].

However, considering dike failure events, countless scenarios are conceivable due to the unpredictability of the breach location alone. The conventional way of premodelling the consequences of events in form of hazard maps can only assess a limited number of scenarios,

\footnotetext{
${ }^{\mathrm{a}}$ Corresponding author: aassm@geomer.de
} 
though. Thus, the high uncertainties render it impossible to ensure that a specific situation is reflected by prepared hazard maps in an acceptable way in case of the occurrence of an event $[4,5]$.

Against this background, our proposition is to model the event-specific inundation when the occurrence is imminent and essential parameters for the model set up are available or procurable. The associated challenge of limited time frames under conditions of the crisis situation and the concurrent need to generate reliable predictions of adequate quality motivate our on-going study of conceptualizing and implementing a workflow that renders operational flood modelling possible for real events.

The aim of this paper, therefore, is to present a precise and practical way of implementing such an operational approach in crisis management for floods.

\subsection{Prospects of operational flood modelling}

Every dike failure constitutes a highly unique situation which is characterized by the respective extent of inundation and distribution of water level. In this respect, the fundamental prospect of operational flood modelling is the capability to reflect and forecast the current, specific situation of imminent or occurring events.

As indicated initially, a high number of scenarios would need to be pre-calculated as a preparation for dike failure events. However, the calculation is reasonable for only a certain number of scenarios as costs and computation time increase with every simulation run. Furthermore, prepared maps often display only the maximum flood extent and inundation depths. Given the high flow velocities related to dike breaches, however, assessing flood progress is of fundamental importance regarding emergency services and evacuation [6].

An additional, significant prospect is the opportunity to assess the effect of potential or planned intervention measures. These directly influence the development of flood progress as means of redirecting the inundation. Regarding the enormous water masses, the implementation of such measures can have unexpected, possibly adverse effects on the progressing inundation. A supposable example would be the unintentional redirection of the inundation to vulnerable areas in the attempt of protecting other exposed elements. Integrating intervention measures in the operational simulation allows to forecast such effects and, thereby, enables more comprehensively informed decision making.

\subsection{Previous work}

Innumerable attempts have been made to assess the hazard of inundation resulting from dike breaches. The majority of hazard assessments are based on defined breach locations and widths which are derived either from historical information of hypothetical assumptions, i.e. scenarios [7].

Commonly, for the assessment of flood development in the hinterland of dike failures, hydrodynamic numerical models are used. Herein, the approach depends mainly on the complexity and the extent of the affected area. Most model approaches are designed and applied for scenario-based or historic event analyses, though, and not intended for operational use [8-10].

An early operational approach has been developed with the model FLUMORE, which was designed for simulating dike breach-induced inundation along the river Rhine in the German state of Baden-Württemberg. It was considered appropriate for operational flood prognoses, mainly considering the restraining factor of computation time. Processing is based on input data with resolutions not exceeding $50 \mathrm{~m} \mathrm{[11].} \mathrm{However,} \mathrm{the} \mathrm{developed}$ workflow was based on external services and not transferable to other regions without model adjustments. Furthermore, to our knowledge the model itself is no longer available.

The model FloodArea HPC [12], deployed for the approach presented in this paper, has recently been used to investigate the potential of running hydrodynamic simulations of dike failure events operationally. The study undertaken in the frame of the EU project IncREO (Increasing Resilience through Earth Observation) has shown that the mere flood simulation is feasible under operational conditions and holds the potential to significantly strengthen flood risk management in crisis situations [13].

Given the feasibility of operational flood modelling, experiences from the Elbe flood in 2013, where two major dike breaches occurred, raised the question of how to effectively implement the process as support in flood crisis management. Following, this topic was comprehensively addressed in the frame of a project commissioned by the flood and water authority of Saxony-Anhalt (LHW), Germany [14]. Findings from previous studies and the on-going research were used to install an operational modelling unit for flood crisis management in Saxony-Anhalt.

In this context, the focus of this paper are the setup and requirements for a profound implementation of an operational modelling unit, fully integrated in flood crisis management and therefore fundamentally supporting decision making in crisis situations.

\section{Operational flood simulation}

In the frame of previous work a comprehensive methodology of simulating flood events operationally has been developed [13].

The modelling and simulation of the inundation process resulting from dike failure offers the possibility to assess flooding depths as well as flow velocities, which is a decisive advantage compared to the assessment of inundation areas, e.g., via aerial imagery. Particularly through the breaching of dikes during fluvial flooding, extreme flow velocities and highly dynamic flood progress prevail. This highlights the benefit of simulation products under operational conditions. Moreover, FloodArea HPC can generate model output at userdefined simulation intervals, which enables to forecast flood progress in high temporal resolution. An additional 
advantage of FloodArea HPC is the robustness of the model against interruptions, abortions and similar incidents. The calculation can be continued at any time based on previous output as well as an automatically generated backup. The generated output up to the time of interruption is already available as result.

The crucial factor in simulating the inundation during the event is the time required for the computation, which is controllable to a large degree, though, via the parametrization of the model. It is, hence, essential to balance increasing computation time against the best quality of results procurable in the given time frame. It is therefore necessary to be able to at least roughly assess expected computation times. Besides the given hardware, operating system and running software, the computation time mainly depends on the terrain character and the parametrization of the model. The most significant influence comes from the size of the inundated area and the pixel size of the input raster. In this respect, the specifications for a simulation under operational conditions should foremost be determined via the required reaction time. The operational guidelines developed in the frame of this study (cf. section 3.3) contain a simple lookup table which helps derive an estimation of simulation time for specified model parameters.

A central strength of the model setup developed with FloodArea HPC is the transferability to various geographical regions on the sole condition that terrain data of adequate resolution is available. Generally, the availability of data as well as the compilation and preprocessing of the databasis are constitutive aspects of rendering operational flood simulation possible and are therefore addressed subsequently.

\subsection{Preliminary work}

Availability of data and a prepared model are essential for the feasibility of operational modelling. A course of action where required data is compiled and processed accurately during the crisis situation is not realistic.

The required input data usually comprise large amounts of data, especially for high-resolution terrain information. Thus, processing times for the preparation can easily extend to several hours. Moreover, the preparation process is complex and, hence, prone to error. This might be evaded by fully automized processes. Here, however, experience shows the risk that prepared scripts might become outdated after few years and require at least minor modifications, which are preferably not undertaken under operational conditions.

Therefore, all required data should be prepared as basic datasets for operational simulations in crisis situations. The readily available datasets should comprise digital terrain models (DTMs) of various spatial resolutions (e.g. $5 \mathrm{~m}$ and $10 \mathrm{~m}$ ), landuse and roughness information in similar resolution, and up-to-date information on existent dikes. As to flood risk management, in order to consider elements of risk a dataset needs to be available accordingly.

The preparation of input data and model setup for potentially affected areas are, thus, a significant prerequisite in order to enable access on a functioning system in case of an imminent event. As processing and preparing data adequately is necessary also for the conduction of scenario-based modelling as a preparation for dike failure events, this general necessity does not pose a disadvantage of the operational approach.

\subsection{Pre-processing of input data}

Especially terrain and landuse information can be prepared as DTMs and corresponding roughness coefficients for use as input during crisis operations. Particular care should be taken in the processing of the terrain data, as the process is rather complex and the DTM character greatly influences the quality of results.

When, as proposed, resampling a DTM of high resolution to lower pixel sizes, the application of a systematic hydrological resample [13] can allow to fully maintain the essential terrain information as given with the higher spatial resolution. The controlling factor is the transfer of hydrologically relevant structures of primarily linear shape to coarser resolutions. Such structures are, for example, constructions in the stream network and the network itself as well as flow impeding constructions, such as railway embankments or elevated roads.

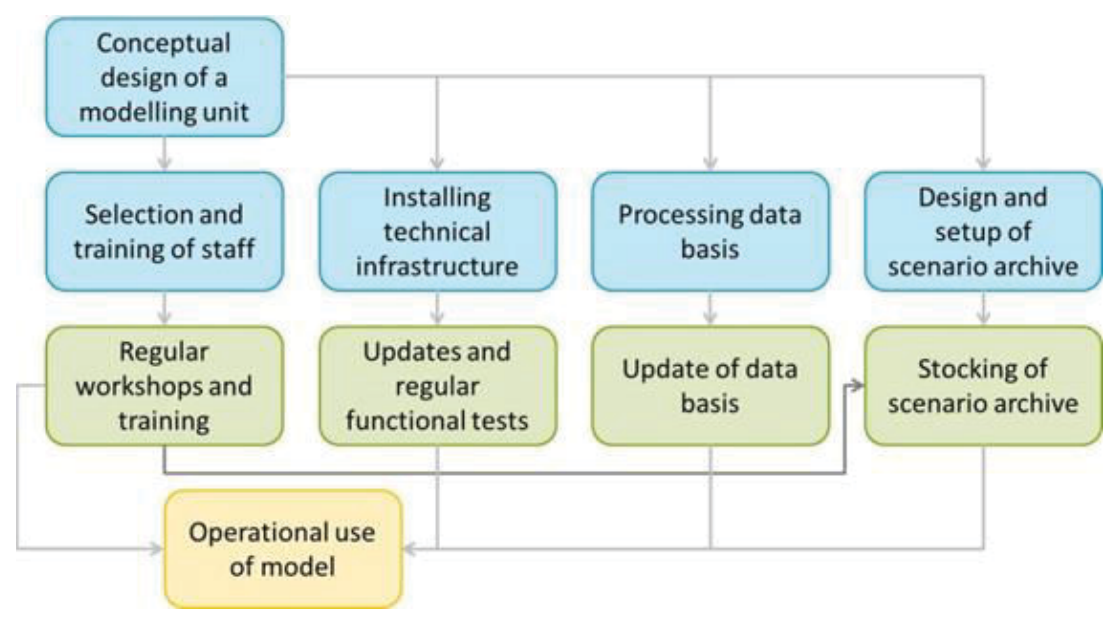

Figure 2. Scheme for establishing an operational modelling unit. 


\section{Operational flood modelling unit}

The objective aimed at in integrating an operational flood modelling unit is to fundamentally support crisis management in flood situations. However, the unit cannot be considered only to be applied individually during a specific crisis situation. Rather must the modelling unit be established comprehensively in the routine of flood management in order to fully function in severe crisis situations. Subsequently, the developed setup for establishing such a unit is introduced.

\subsection{Setup}

The approach for setting up an operational flood modelling unit is schematically illustrated in Fig. 2. Therein, four central divisions are displayed with the selection and training of staff, installing technical infrastructure, processing of the data basis and the design and setup of a scenario archive.

\subsubsection{Staff}

The staff of a modelling unit should meet a certain profile of qualification, i.a. professional background in natural or engineering sciences with a focus on water management as well as practical experience with geographic information systems, hydraulic modelling and especially the specific, deployed model. Once the team has been instated, steady training is inevitable. In the frame of training, the simulation workflow should also be applied under operational conditions, e.g. in the form of regular exercises.

\subsubsection{Technical infrastructure}

In terms of the technical setup some critical aspects need to be kept in mind, as the establishment of hardware and software always bears the risk of becoming outdated. One possibility of maintaining the established systems in their setup state would be to shield them from any network connections. However, this includes the consequence of the working systems drifting apart as technical innovations are not taken on. Also, access to data is not possible via a network.

Considering this, we propose that all computers remain connected to the network while disabling all automatic updates. This setup needs to be arranged in agreement with the network administration. All operating system updates should be conducted by the users promptly on notification through the system, provided that no critical flood situation is imminent. All updates must be followed by a basic test of all necessary functionalities, i.e. the geographic information system, the simulation software and the license dongle. The same procedure accounts for virus protection scanning.

Updates of the geographic information system and the simulation software take place linked to workshops and exercises. This permits to directly identify potential problems by implicitly conducting a comprehensive test. According to the requirements, problems can then be fixed directly or the previous system will temporarily be restored in order to keep up the permanent functionality.

All hardware used for the simulation as well as data servers containing base data and simulation results require emergency power supply and overvoltage protection.

\subsubsection{Processing of the data basis}

The fundamental aspects on the data basis and respective processing have already been outlined previously in the sections on preliminary work and preprocessing of input data for operational flood simulation (cf. section 2.1, 2.2).

Further task of the modelling unit is to keep the data basis up to date and revise data in respect of changes and alterations, for example, in the real surface or regarding infrastructure.

\subsubsection{Scenario archive}

The results of calculated scenarios, which are produced in the course of exercises, should be archived in

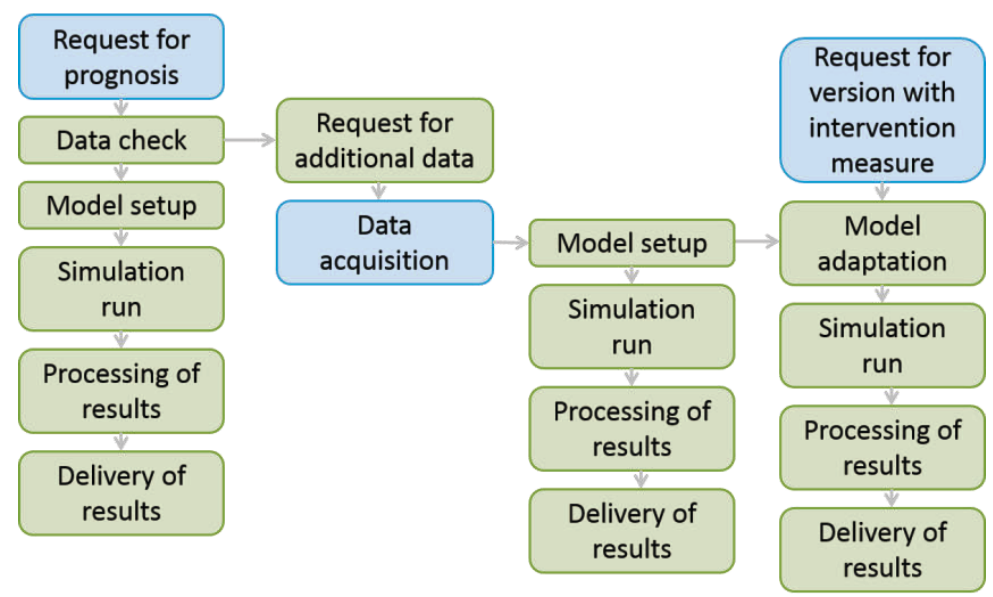

Figure 3. Schematic workflow of a modelling operation. In the course of the crisis simulation runs can branch off into variations in parametrization or input information. 
a clear, manageable structure. In this way, during real event situations such archived results are readily available and can serve for an initial overview, equivalent to conventional hazard maps. An elaborated proposal for an archive structure is contained in the developed operational guidelines (cf. section 3.3).

\subsubsection{Operational modelling workflow}

Once a modelling unit has been set up according to the outlined concept, it can operate during crisis situations according to a powerful as well as flexible workflow (Fig. 3).

When an event is imminent or occurring, an initial simulation is started based on readily available data. An additional simulation can be initiated. Subsequently, a branching of calculation runs is possible, for instance in order to test the effect of potential measures. In this case, one branch would simulate the inundation without the intervention measure, the other branch simulates the influenced flood progress. Up to the point, at which the measure becomes effective, only one simulation run is necessary. The possibility to continue calculations from arbitrary points of time in the simulation is one of the functionalities of FloodArea HPC.

\subsection{Requirements under operational conditions}

In order for an instated flood modelling unit to be able to support crisis management to a relevant degree, several requirements need to be met.

Concerning resources, the following conditions have been elaborated:

Regarding software and hardware two congruent systems are necessary, if only for reasons of reliability and failure safety. The possibility to model multiple scenarios parallely, besides, requires parallely running systems. We therefore propose an infrastructure of four fully equipped simulation systems (i.e. computers and software). A higher number of parallel simulation runs seems improbable and imposes fairly high demands on the simulation management. However, the possibility of two simultaneous dike breaches must not be neglected. The proposed equipment covers handling two dike breaches with two versions respectively.

Regarding modelling staff, two persons for the operation of the flood modelling should be available at all times. In this configuration, each persons is in charge of two systems, i.e. scenarios.

Beyond this basic setup, the most crucial requirements are relating to the integration in flood crisis management. Here, specifically defined requests and clear communication are fundamental for the modelling unit to operate and function in an effective and supportive way. Foremost, it needs to be clarified what format results should be delivered in and to what degree they are to be interpreted cartographically and with regards to content. Miscommunications of this kind can easily jeopardize the supporting potential of operationally generated prognoses.
A crucial specification for requested prognosis during a given crisis event is the point in time the simulation results need to be made available and the prediction period that is requested by that time. Particularly for situations with fast and extensive flood progress, as resulting from dike failure events, computation time rises exponentially with increasing expansion of the inundation area. At the same time, uncertainties in the prediction augment with prediction periods reaching further into the future. Therefore, the urgency of simulating very long periods in the frame of the first time limit should be reasonably prioritized and it is advisable to schedule several deadlines for delivering simulation results. Furthermore, critical infrastructure and elements in the inundation area need to be communicated.

On the other hand, certain requirements derive also from the side of the modelling unit. During the course of an event the acquisition and provisioning of information and data is essential for the task of simulating the imminent inundation development. Event-specific parameters comprise the time and location of a dike breach, breach width, water level information and land use information particularly in the area of the breach. Activities such as daily flood extent mapping and measurements of flow through the breach significantly support the performance of flood modelling. E.g., a checklist can help ensure that requested data and information is collected and delivered by responsible parties.

In order to facilitate operational procedure and ensure the consideration of fundamental criteria, operational guidelines have been elaborated and designed, which are presented subsequently.

\subsection{Operational guidelines}

The operational guidelines are an extract of the operational modelling approach for inundation from dike breaches and intended to serve as assistance during operational activity. Thus, the guidelines are designed to be completed and continued, e.g., with references to contacts or locations of corresponding lists. Newly acquired knowledge and experiences should be considered as well. The individual guidelines and checklists currently comprise:

- A schematic overview of the integration of modelling operations in staff activity: The scheme illustrates the basic tasks and essential procedures of responsible staff units.

- A schematic overview of the modelling workflow (cf. Fig. 3)

- Relevant information for prognosis requests: The bullet list supports the modeler in determining the simulation approach

- Checklist for the acquisition and compilation of event-specific data: The list helps document information on the breach location, breach characteristics, water levels and utilized data sources.

- Instructions regarding the photographic documentation of the breach location corresponding the model- 
ling requirements also for persons outside the area of expertise

- Notification form for the documentation of eventspecific data: This form accompanies the records of the event $\log$ with a focus on information relevant for modelling and respective results. It is furthermore an important basis for the validation of modelling results.

- Checklist for the determination of model parameters: Using this list, based on the event-specific flood plain characteristics and the available time frame the variable model parameters can be determined.

- Checklist for reviewing the data basis for the simulation: All available data for the simulation area and the close range around the breach should be listed, checked and, if required, modified

- Instructions for the interpretation of remote sensing imagery for generating a flood mask

- Classification form for assessing and describing the quality of model results: Dependent on the conditions of modelling and the available data basis, the modelling results are assigned to different levels of quality. This helps to evaluate results as support for decision making

- Product list for the delivery of generated products: This list serves for the documentation of delivered data and formats

- Layout templates: ArcGIS templates, e.g., ensure a clear and consistent layout of the various modelling results

- Directory structure of simulation data: Recommendation for storing and archiving simulation data

According to this concept, the operational guidelines support the unit to efficiently manage and at the same time document the complex procedures under the extreme, critical conditions of operational modelling.

\section{Conclusions and outlook}

Operational modelling with the possibility to implement real-event parameters poses the opportunity to enhance prognoses in case of the occurrence of an event and by this allows both for higher preparedness and increased coping capacity.

The flood simulation itself is feasible under operational conditions and the technology, foremost regarding the model, is globally applicable.

However, as the presented operational modelling configuration focusses on the application for large dike failure events, this implies treating with large datasets, which poses a significant challenge with highly critical operational conditions concerning mostly processing times.

A further, practical challenge of operational simulation are inundation areas stretching over borders, such as federal state boundaries. The situation of data being available only state wide, as is e.g. in Germany, has not yet been overcome. For large rivers, the potential of events that result in cross-state inundation is implicit and the data basis must be expanded respectively.

Challenges in the context of framework conditions will arise regarding the breach and flood monitoring process. An approach gaining prominence with advances in drone and microcopter technology is the acquisition of real-time information, e.g. regarding the breach itself, from aerial imagery and subsequent analysis, such as the derivation of geometrical parameters [15].

It needs to be noted that an operational simulation always entails a compromise among reaction time and level of detail. The quality of results generated in the frame of an engineering modelling process without pressure of time will not be achieved. Nonetheless, with the setup and workflows elaborated in this paper, the implementation and operation of a flood modelling unit for crisis situation is feasible and can function reliably. When a functioning system is prepared, operationally produced modelling results significantly support disaster management in informed decision-making and thereby contribute to the mitigation of severe disaster situations as posed by dike failure events.

\section{Acknowledgements}

The presented study has to great part been undertaken in the frame of the project "Erarbeitung einer Methodik zur zeitnahen Abschätzung von Überflutungssituationen infolge von Deichbrüchen unter Berücksichtigung der Erfahrungen im Juni-Hochwasser 2013 an der Elbe" commissioned by the flood and water authority of Saxony-Anhalt (LHW), Germany. The project was carried out in cooperation with UNIWASSER GmbH (lead), Kaiserslautern, and Magdeburg-Stendal University.

Part of the research was carried out in the frame of the IncREO (Increasing Resilience through Earth Observation) project funding from the European Community's Seventh Framework Programme under grant agreement $\mathrm{n}^{\circ} 312461$.

\section{References}

1. Bieberstein, A., et al. (2007). River dikes actual developments for improvement in flood safety. Bautechnik, 84(12), pp. 829-837.

2. Lind, N., Pandey, M. \& Nathwani, J. (2009). Assessing and affording the control of flood risk. Structural Safety, 31(2), pp. 143-147.

3. European Parliament and Council (2007). Directive 2007/60/EC on the assessment and management of flood risks. Official Journal of the European Union, L 288, pp. 27-34.

4. Bouwer, L.M., et al. (2009). Inundation scenarios for flood damage evaluation in polder areas. Natural Hazards and Earth System Sciences, 9(6), pp. 1995-2007.

5. Vorogushyn, S., et al. (2010). A new methodology for flood hazard assessment considering dike breaches. Water Resources Research, 46(8), pp. 17. 
6. CIRIA (2013). The International Levee Handbook. CIRIA, London, pp. 1332.

7. Vorogushyn, S. (2008). Analysis of flood hazard under consideration of dike breaches. Dissertation, University of Potsdam, pp. 165.

8. Cannata, M. \& Marzocchi, R. (2012). Twodimensional dam break flooding simulation: a GIS-embedded approach. Natural Hazards, 61(3), pp. 1143-1159.

9. Jonkman, S.N., Kok, M. \& Vrijling, J.K. (2008). Flood Risk Assessment in the Netherlands: A Case Study for Dike Ring South Holland. Risk Analysis, 28(5), pp. 1357-1374.

10. Lin, B., Wicks, J. \& Falconer, R.A. (2006). Integrating $1 \mathrm{D}$ and 2D hydrodynamic models for flood simulation. Water Management, 159(1), pp. 19-25.

11. Homagk, P. \& Ludwig, K. (2009). Operational forecast of flooding as a consequence of rhine dykebreaks in Baden-Württemberg. WasserWirtschaft, 99(1/2), pp. 12-16.

12. geomer GmbH \& RZB GbR (2015). FloodArea and FloodAreaHPC: ArcGIS extension for calculating flooded areas. geomer $\mathrm{GmbH}$, Heidelberg, pp. 67.

13. Anders, K., Assmann, A. \& Fritsch, K. (2014). Operationelle Überflutungssimulation für Deichbrüche. AGIT - Journal für Angewandte Geoinformatik, 1, pp. 228-233.

14. Uniwasser GmbH, geomer $\mathrm{GmbH} \&$ Magdeburg-Stendal, H. (2015). Abschlussbericht: Erarbeitung einer Methodik zur zeitnahen Abschätzung von Überflutungssituationen infolge von Deichbrüchen unter Berücksichtigung der Erfahrungen im Juni-Hochwasser 2013 an der Elbe. Landesbetrieb für Hochwasserschutz und Wasserwirtschaft Sachsen-Anhalt, unpublished, p. 110.

15. Jüpner, R., Brauneck, J. \& Pohl, R. (2015). Einsatz von Drohnen im Hochwasserfall Erfahrungen und Ideen. WasserWirtschaft, 9, pp. 49-54. 